
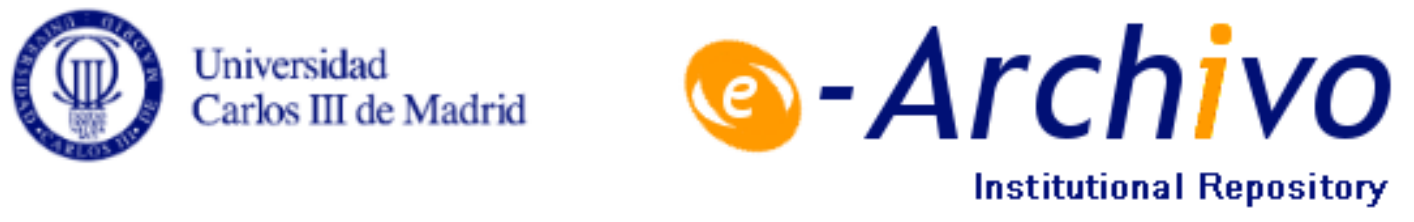

This is a postprint version of the following published document:

Robinson, James C; Sánchez-Gabites, Jaime J. (2016). On finite-dimensional global attractors of homeomorphisms. Bulletin of the London Mathematical Society, vol. 48, n. 6, pp. 483-498. Avalaible in https://doi.org/10.1112/blms/bdw011.

(c) Wiley 


\title{
On finite-dimensional global attractors of homeomorphisms
}

\author{
James C. Robinson and Jaime J. Sánchez-Gabites
}

\begin{abstract}
Let $E$ be a normed linear space and suppose that $\mathbb{A}$ is the global attractor of either (i) a homeomorphism $F: E \rightarrow E$ or (ii) a semigroup $S(\cdot)$ on $E$ that is injective on $\mathbb{A}$. In both cases, $\mathbb{A}$ has trivial shape, and the dynamics on $\mathbb{A}$ can be described by a homeomorphism $F: \mathbb{A} \rightarrow \mathbb{A}$ (in the second case we set $F=S(t)$ for some $t>0)$. If the topological dimension of $\mathbb{A}$ is finite, then we show that for any $\epsilon>0$ there is an embedding $e: \mathbb{A} \rightarrow \mathbb{R}^{k}$, with $k \sim \operatorname{dim}(\mathbb{A})$, and a (dynamical) homeomorphism $f: \mathbb{R}^{k} \rightarrow \mathbb{R}^{k}$ such that $F$ is conjugate to $f$ on $\mathbb{A}$ (that is, $\left.F\right|_{\mathbb{A}}=e^{-1} \circ f \circ e$ ) and $f$ has a global attractor $A_{f}$ with $e(\mathbb{A}) \subseteq A_{f} \subseteq N(e(\mathbb{A}), \epsilon)$. In other words, we show that the dynamics on $\mathbb{A}$ are essentially finite dimensional.

We characterise subsets of $\mathbb{R}^{n}$ that can be the global attractors of homeomorphisms as cellular sets, give elementary proofs of various topological results connected to Borsuk's theory of shape and cellularity in Euclidean spaces, and prove a controlled homeomorphism extension theorem. We also show that we could achieve $e(\mathbb{A})=A_{f}$ under the assumption of a stronger controlled homeomorphism extension theorem.
\end{abstract}

\section{Introduction}

One can recast many of the important equations of mathematical physics within the framework of infinite-dimensional dynamical systems, that is, dynamical systems evolving in an infinitedimensional phase space. The theory of such systems has been systematically developed over the last three decades, and is well covered in the monographs by Babin and Vishik [1], Chepyzhov and Vishik [5], Chueshov [6], Hale [15], Ladyzhenskaya [20], Robinson [29], and Temam [32]. One of the most striking results in this theory is that in many interesting examples the long-time dynamics can be captured by a finite-dimensional subset of the ambient (infinite-dimensional) phase space, the 'global attractor'.

However, this statement says nothing a priori about the dynamics restricted to the attractor, and it is natural to ask in what sense (if any) these dynamics are themselves finite dimensional. This question was first posed in this generality by Eden et al. [9], and subsequently discussed by Robinson [28] and Romanov [31]. Ideally one would construct a finite-dimensional ordinary differential equation (ODE) whose dynamics reproduce those on the attractor. This is certainly possible if the original system possesses an inertial manifold [11], but the existence of such an object requires restrictive conditions (a 'spectral gap condition' on the linear part of the equation) that prevent the theory being applicable to many important examples, such as the two-dimensional Navier-Stokes equations.

However, the construction of such an ODE seems very difficult, essentially because it would require a bi-Lipschitz embedding of the attractor into a Euclidean space (this can be slightly weakened to allow logarithmic corrections, see $[\mathbf{2 7}]$ ). An intrinsic characterisation of sets that admit such an embedding is a major open problem in the theory of metric spaces (see [16], for example), and there are examples due to Eden et al. [10] that show that such an embedding

Sánchez-Gabites was supported by a MICINN grant (MTM 2009-07030). 
(even with a logarithmic correction) is in general not possible for the attractors of infinitedimensional dynamical systems.

Thus in this paper we aim to reproduce not the continuous dynamics on the attractor, but the discrete dynamics that come from considering the time $T$ map of the flow, for some fixed $T>0$. In fact, we will consider the discrete problem from the outset, in which case the attractor arises from the iteration of some given homeomorphism.

At the heart of our construction is a classical theorem due to Menger [23] a n d N ${ }^{*}$ obeling $[\mathbf{2 5}]$ (see also $[\mathbf{1 7}, \mathbf{3 0}]$ ), which guarantees that any finite-dimensional compact metric space can be embedded into a finite-dimensional Euclidean space of comparable dimension. The following theorem states this more precisely.

Theorem 1.1 (Menger-Nöbeling). Let $(X, d)$ be a compact metric space of dimension no larger than $d$. Then the set of all homeomorphisms of $X$ onto a subset of $\mathbb{R}^{2 d+1}$ is dense in $C^{0}\left(X, \mathbb{R}^{2 d+1}\right)$. (In fact, the homeomorphisms form a dense $G_{\delta}$ in $C^{0}\left(X, \mathbb{R}^{2 d+1}\right)$.)

We use this theorem to make a homeomorphic copy $X$ of our original finite-dimensional attractor, along with its dynamics, into some $\mathbb{R}^{k}$. The main task is to find a way to extend the embedded dynamics from $X$ onto the whole of $\mathbb{R}^{k}$, and to make the set $X$ an attractor (or as nearly as possible) for the resulting dynamics.

In Section 2, we show that cellularity characterises the global attractors of homeomorphisms in Euclidean spaces, based in part on previous work of Garay [12]. In Section 3, we show that global attractors of homeomorphisms, and of continuous time semigroups, have trivial shape, a property that is topologically invariant. We recall in Section 4 the cellularity criterion of McMillan [22], which allows us to boost a set with trivial shape to one that is cellular by adding an extra dimension to the ambient space. In Section 5, we prove a theorem that provides a controlled extension of a homeomorphism from a compact subset of $\mathbb{R}^{k}$ to a map on $\mathbb{R}^{2 k}$, based on a trick of Klee [19]. Finally, we combine these techniques in Section 6 to show that discrete dynamics on finite-dimensional global attractors are no more complicated than the dynamics on global attractors of homeomorphisms in finite-dimensional spaces.

However, the main result of the paper (Theorem 6.1) does not produce a homeomorphism that has the desired set $X$ as its global attractor, but only as a subset of the global attractor. In the final section, we show that the set $X$ itself could be made the global attractor given a certain controlled homeomorphism extension theorem that (in some sense) 'respects the cellularity of $X$ '. Details are given in Proposition 7.1.

\section{Global attractors and cellularity}

\subsection{Global attractors in normed spaces}

Suppose that $E$ is a normed space, and that $F: E \rightarrow E$ is a continuous map. Then $\mathbb{A} \subseteq E$ is a global attractor for $F$ if

(1) $\mathbb{A}$ is compact;

(2) $\mathbb{A}$ is invariant, that is, $F(\mathbb{A})=\mathbb{A}$; and

(3) $\mathbb{A}$ attracts bounded sets, that is, for every bounded subset $B$ of $E$,

$$
\operatorname{dist}\left(F^{n}(B), \mathbb{A}\right) \rightarrow 0 \quad \text { as } n \rightarrow \infty,
$$

where $\operatorname{dist}(A, B)=\sup _{a \in A} \inf _{b \in B}\|a-b\|$.

Note that if the global attractor exists, then it is unique and is the minimal closed set that attracts bounded sets (both follow from the fact that if $Z$ attracts bounded sets, then $\operatorname{dist}(\mathbb{A}, Z)=\operatorname{dist}\left(F^{n}(\mathbb{A}), Z\right) \rightarrow 0$ as $\left.n \rightarrow \infty\right)$. 
Condition (3) is equivalent to requiring that for any bounded set $B$ of $E$ and every $\epsilon>0$ there exists $n_{0}$ such that $F^{n}(B) \subseteq N(\mathbb{A}, \epsilon)$ whenever $n \geqslant n_{0}$. Here $N(\mathbb{A}, \epsilon)$ denotes the set $\{x \in E: \operatorname{dist}(x, \mathbb{A})<\epsilon\}$. In particular, this implies that for any bounded set $B$ containing $\mathbb{A}$, the equality $\mathbb{A}=\bigcap_{n} F^{n}(B)$ holds.

The existence of a global attractor is equivalent to the existence of a compact attracting set $K$, that is, a compact set $K$ satisfying (3) above in place of $\mathbb{A}$.

Theorem 2.1. The map $F$ has a global attractor $\mathbb{A}$ if and only if it has a compact attracting set $K$, and in this case

$$
\mathbb{A}=\bigcap_{j=0}^{\infty} F^{j}(K)
$$

Proof. It is relatively straightforward to show that given the existence of a compact attracting set $K$, for any bounded set $B$ the set

$$
\begin{aligned}
\omega(B) & :=\bigcap_{k \geqslant 0} \overline{\bigcup_{n \geqslant k} F^{n}(B)} \\
& =\left\{x \in E: x=\lim _{j \rightarrow \infty} F^{n_{j}}\left(b_{j}\right), n_{j} \rightarrow \infty, b_{j} \in B\right\}
\end{aligned}
$$

is a subset of $K$ that is compact, invariant, and attracts $B$ (see [15], or [29, Theorem 11.3], for example). We now show that $\omega(K)$ is the global attractor. Consider

$$
\mathbb{A}=\overline{\bigcup_{B \text { bounded }} \omega(B)} \text {. }
$$

Since $\omega(B) \subseteq K$ for every bounded $B$, this is a closed subset of $K$, and so compact. It is clearly invariant since every $\omega(B)$ is invariant, and it attracts every bounded set, so it must be the global attractor since this is unique. It is immediate from $(2.3)$ that $\omega(K) \subseteq \mathbb{A}$. Since $\mathbb{A}$ is the minimal closed set that attracts bounded sets, $\mathbb{A} \subseteq K$ and hence $\mathbb{A}=\omega(\mathbb{A}) \subseteq \omega(K)$.

Finally, to show that (2.1) holds, note that $\omega(K) \subseteq K$ and then, since $\omega(K)$ is invariant,

$$
\omega(K) \subseteq F_{k \geqslant 0}^{k}(K)
$$

and it follows from (2.2) that

$$
F^{k}(K) \subseteq \overline{\bigcup_{n \geqslant k} F^{n}(K)} \Rightarrow \bigcap_{k=0}^{\infty} F^{k}(K) \subseteq \omega(K),
$$

which yields (2.1).

In a normed space, we write $B_{R}$ to denote the open ball and $\bar{B}_{R}$ the closed ball of radius $R$ and with centre 0 . A closed subset $C$ of a normed space is a cell if $(C, \operatorname{int}(C))$ is homeomorphic to $\left(\bar{B}_{1}, B_{1}\right)$ (evidently the choice of radius 1 in this definition is irrelevant). Note that, as a consequence of the theorem on invariance of domain, if $E$ is finite dimensional, then any subset $C$ of $E$ that is homeomorphic to $\bar{B}_{1}$ is a cell in this sense.

We now show that any global attractor of a homeomorphism must be strongly cellular. A set $X$ is cellular in $E$ if

$$
X=\bigcap_{j=1}^{\infty} C_{j}
$$

for some sequence $\left(C_{j}\right)$ of cells that is strictly decreasing in the sense that $C_{j+1} \subseteq \operatorname{int}\left(C_{j}\right)$ for each $j$. The sequence $\left(C_{j}\right)$ is then called a cellular sequence for $X$. If, in addition, for any open 
set $U$ containing $X$ there is a $j$ such that $C_{j} \subseteq U$, then $X$ is strongly cellular (correspondingly, $\left(C_{j}\right)$ is then said to be a strongly cellular sequence for $X$ ). Cellularity and strong cellularity are equivalent in finite-dimensional spaces because of their local compactness (see $[\mathbf{1 2}, \mathbf{2 1}])$.

Lemma 2.2. If $\mathbb{A}$ is the global attractor of a homeomorphism $F: E \rightarrow E$, where $E$ is a normed space, then $\mathbb{A}$ is strongly cellular.

Proof. Choose $R$ sufficiently large that $\mathbb{A} \subseteq B_{R}$. Since $\mathbb{A}$ is the global attractor, there exists an $n$ such that

$$
F^{n}\left(\bar{B}_{R}\right) \subseteq B_{R}
$$

For each $j=1,2, \ldots$, let $C_{j}=F^{n j}\left(\bar{B}_{R}\right)$. By definition, $F^{n j}$ restricts to a homeomorphism from $\bar{B}_{R}$ onto $C_{j}$. Moreover, since $F^{n j}$ is defined on the whole space $E$, it maps the interior of $\bar{B}_{R}$ onto the interior of $C_{j}$. Therefore, it provides a homeomorphism from $\left(\bar{B}_{R}, B_{R}\right)$ onto $\left(C_{j}, \operatorname{int}\left(C_{j}\right)\right)$, proving that the $C_{j}$ are all cells. The choice of $n$ guarantees that $C_{j+1} \subseteq$ $F^{n j}\left(B_{R}\right)=\operatorname{int}\left(C_{j}\right)$ for each $j$, so the $C_{j}$ form a strictly decreasing sequence.

Clearly,

$$
\mathbb{A} \subseteq \bigcap_{j=1}^{\infty} C_{j}
$$

because $\mathbb{A}$ is invariant. Since $\mathbb{A}$ is the global attractor of $F$, it has to attract the bounded set $\bar{B}_{R}$, so given any open $U \supset \mathbb{A}$ there exists $j$ such that $C_{j} \subseteq U$. This readily implies that

$$
\mathbb{A}=\bigcap_{j=1}^{\infty} C_{j}
$$

so $\mathbb{A}$ is strongly cellular.

\subsection{Global attractors in Euclidean spaces}

We now want to show that any cellular subset of $\mathbb{R}^{n}$ can be the global attractor of some homeomorphism on $\mathbb{R}^{n}$. We start by showing that any cellular set $X$ in $\mathbb{R}^{n}$ is 'pointlike', that is, $\mathbb{R}^{n} \backslash X \simeq \mathbb{R}^{n} \backslash\{0\}$.

Lemma 2.3 (After Brown [4]). If $X \subseteq \mathbb{R}^{n}$ is cellular, then there exists a continuous map $g: \mathbb{R}^{n}$ $\rightarrow \mathbb{R}^{n}$ such that $g^{-1}(\{0\})=X$ and $\left.g\right|_{\mathbb{R}^{n} \backslash X}: \mathbb{R}^{n} \backslash X \rightarrow \mathbb{R}^{n} \backslash\{0\}$ is a homeomorphism. Moreover, if $X \subseteq B_{R}$, then $g$ can be chosen to be the identity outside $B_{R}$.

Proof. Let

$$
X=\bigcap_{j=1}^{\infty} Q_{j},
$$

where each $Q_{j}$ is a cell with $Q_{j+1} \subseteq \operatorname{int}\left(Q_{j}\right)$. Perhaps discarding the first few $Q_{j}$, we can assume that $Q_{1}$ is a subset of the interior of the ball $Q:=\bar{B}_{R}$.

Let $g_{1}: Q \rightarrow Q$ be a homeomorphism such that $g_{1} \mid \partial Q=$ id and $g_{1}\left(Q_{1}\right) \subseteq B_{R / 2}$. To see that such a homeomorphism exists, first note that there exists $0<r<R$ such that $Q_{1}$ is contained in $B_{r}$. Consider a strictly increasing continuous map $a:[0, R] \rightarrow[0, R]$ such that $a(R)=R, a$ is the identity near 0 , and $a(r)=R / 2$. Then the map $g_{1}: Q \rightarrow Q$ given by

$$
g_{1}(x)=a(|x|) \frac{x}{|x|}
$$

is a homeomorphism (the fact that $a$ is the identity near 0 guarantees that $h$ is continuous) such that $g_{1}\left(B_{r}\right)$, and hence also $g_{1}\left(Q_{1}\right)$, are contained in $B_{R / 2}$. 
Now, given $g_{j-1}$, let $g_{j}: Q \rightarrow Q$ be a homeomorphism such that

$$
g_{j}(x)=g_{j-1}(x) \quad \text { for } x \in Q \backslash Q_{j-1} \quad \text { and } \quad g_{j}\left(Q_{j}\right) \subseteq B_{R /(j+1)} .
$$

(That such a homeomorphism exists follows from a similar argument to that given above.)

By the construction, $g_{k}\left|\left(Q \backslash Q_{j}\right)=g_{j}\right|\left(Q \backslash Q_{j}\right)$ and $g_{k}\left(Q_{j}\right)=g_{j}\left(Q_{j}\right)$ if $k \geqslant j \geqslant 1$. Now define a map $g: Q \rightarrow Q$ by setting $g\left|\left(Q \backslash Q_{j}\right)=g_{j}\right|\left(Q \backslash Q_{j}\right)$ if $j \geqslant 1$ and $g(X)=\{0\}$. Then $g \mid \partial Q=$ id, $g \mid(Q \backslash X)$ is an embedding whose image is $g(Q \backslash X)=Q \backslash \bigcap_{j=1}^{\infty} g_{j}\left(Q_{j}\right)=Q \backslash\{0\}$, and $g$ is continuous at the points of $X$ as $g\left(Q_{j}\right) \subseteq B(0, r /(j+1))$ if $j \geqslant 1$. Finally, extend $g$ to $\mathbb{R}^{n}$ by letting $g$ be the identity on $\mathbb{R}^{n} \backslash Q$.

Using this, we can show that any cellular $X \subseteq \mathbb{R}^{n}$ is the global attractor of some homeomorphism. In fact, one can use almost exactly the same proof to define an abstract flow on $\mathbb{R}^{n}$ (even a smooth one generated by an ODE) that has $X$ as the global attractor; for details on these refinements see $G$ " unther and Segal [14], G"unther [13], and Pinto de Moura et al. [27].

Theorem 2.4 (After Theorem 2.7 in Garay [12]). If $X$ is a cellular subset of $\mathbb{R}^{n}$, then there exists a homeomorphism $h: \mathbb{R}^{n} \rightarrow \mathbb{R}^{n}$ such that $h(x)=x$ for all $x \in X$ and $X$ is the global attractor for the dynamical system generated by $h$. Moreover, if $X \subseteq B_{R}$, then there exists a constant $\rho>0$ such that $h\left(B_{r}\right) \subseteq B_{r-\rho}$ for every $r \geqslant R$.

Proof. After rescaling, we may assume without loss of generality that $R>1$. Choose $R>{ }^{\prime}>$ 1 such that $X \subseteq B_{R^{\prime}}$ and let $g: \mathbb{R}^{n} \rightarrow \mathbb{R}^{n}$ be the map given by Lemma 2.3 , with the property that $g$ is the identity outside the ball $B_{R^{\prime}}$. Consider the annuli

$$
R_{k}=\left\{x \in \mathbb{R}^{n}: 2^{-(k+1)} \leqslant|x| \leqslant 2^{-k}\right\}
$$

for $k=0,1,2, \ldots$ Each $R_{k}$ is a compact subset of $\mathbb{R}^{n} \backslash\{0\}$, and so $g^{-1}: \mathbb{R}^{n} \backslash\{0\} \rightarrow \mathbb{R}^{n} \backslash X$ is uniformly continuous on each set $R_{k} \cup R_{k+1}$; in particular, there exists a $b_{k}$ such that if $x, y \in R_{k} \cup R_{k+1}$ with $x=r \xi$ and $y=s \xi$, for some $\xi$ with $|\xi|=1$, then

$$
\left|g^{-1}(r \xi)-g^{-1}(s \xi)\right| \leqslant 2^{-k}
$$

provided that $|r-s| \leqslant b_{k}$. Redefine $b_{k}$ (if necessary) to ensure that $R-b_{0}>R^{\prime}$ and

$$
b_{k}<\min \left(b_{k-1} / 2,2^{-(k+3)}\right),
$$

and let $\beta:[0, \infty) \rightarrow[0, \infty)$ be the function with $\beta(0)=0, \beta\left(2^{-k}\right)=b_{k}$ for each $k \in \mathbb{N}$, $\left.\beta\right|_{\left[2^{\left.-(k+1), 2^{-k}\right]}\right.}$ affine for each $k \in \mathbb{N}$, and $\beta(r)=b_{0}$ for $r \geqslant 1$. Now set $\alpha(r)=r-\beta(r)$ and note that (i) $\alpha(0)=0, \alpha(r) \rightarrow \infty$ as $r \rightarrow \infty$, and $\alpha:[0, \infty) \rightarrow[0, \infty)$ is strictly increasing, so $\alpha$ is a homeomorphism; (ii) $\alpha^{k}(r) \rightarrow 0$ as $k \rightarrow \infty$ for any $r>0$; and (iii) $|r-\alpha(r)|=\beta(r) \rightarrow 0$ as $r \rightarrow 0$. For $x \notin X$, let

$$
h(x)=g^{-1}\left[\alpha(|g(x)|) \frac{g(x)}{|g(x)|}\right]
$$

and for $x \in X$ set $h(x)=x$. Clearly, $X$ is the global attractor of this homeomorphism, since

$$
g\left[h^{k}(x)\right]=\alpha^{k}(|g(x)|) \frac{g(x)}{|g(x)|},
$$

$\alpha^{k}(r) \rightarrow 0$ as $k \rightarrow \infty$, and $\operatorname{dist}(y, X) \rightarrow 0$ as $g(y) \rightarrow 0$. 
This mapping satisfies the requirements of the theorem; the only possible issue is continuity at each $x \in X$. First observe the following: if $y \in g^{-1}\left(R_{k}\right)$, then $y=g^{-1}(r \xi)$ for some $r, \xi$ with $2^{-(k+1)} \leqslant r \leqslant 2^{-k}$ and $|\xi|=1$, and so by $(2.4)$

$$
|y-h(y)|=\left|g^{-1}(r \xi)-g^{-1}(\alpha(r) \xi)\right| \leqslant 2^{-k},
$$

since $|r-\alpha(r)|=\beta(r) \leqslant b_{k}$, and thus also $\alpha(r) \geqslant 2^{-(k+2)}$ implying that $\alpha(r) \xi \in R_{k} \cup R_{k+1}$. Now fix $x \in X$ and $\epsilon>0$. Choose $N>0$ such that $2^{-N}<\epsilon / 2$ and $0<\delta<\epsilon / 2$ so small that $|y-x|<\delta$ implies $y \in X$ or $y \in g^{-1}\left(R_{k}\right)$ for some $k \geqslant N$. Then if $|y-x|<\delta$

$$
|h(x)-h(y)| \leqslant|x-y|+|y-h(y)| \leqslant \epsilon / 2+2^{-N}<\epsilon,
$$

which shows that $h$ is continuous.

Finally, pick $x \in \mathbb{R}^{n}$ with $|x|=r \geqslant R$. Then $g(x)=x$ because $g$ is the identity outside $B_{R}$, and $\beta(|x|)=b_{0}$ because $R$ was assumed to be bigger than 1 . Thus

$$
\alpha(|g(x)|) \frac{g(x)}{|g(x)|}=\left(|x|-b_{0}\right) \frac{x}{|x|}=\left(r-b_{0}\right) \frac{x}{|x|}
$$

has modulus $r-b_{0} \geqslant R-b_{0}>R^{\prime}$, so $g^{-1}$ leaves it fixed. Consequently, $h$ transforms the sphere of radius $r$ onto the sphere of radius $r-b_{0}$, and therefore $h\left(B_{r}\right) \subseteq B_{r-b_{0}}$.

Note that the results of Lemma 2.2 and Theorem 2.4 show that cellularity is the characteristic feature of global attractors of homeomorphisms in Euclidean spaces.

In comparison with the results in the paper by Garay [12], we note that his definition of a global attractor $X$ (a 'globally asymptotically stable compact invariant set' in his terminology) requires $X$ to be asymptotically stable and to attract points of $E$. If $E$ is finite dimensional, then this notion of attractor coincides with the one used in this paper, but this is no longer true when $E$ is infinite dimensional.

\section{Global attractors have trivial shape}

\subsection{Global attractors of homeomorphisms}

Given a finite-dimensional global attractor $\mathbb{A}$ of a homeomorphism on some normed space $E$, our aim is to construct a homeomorphism on some $\mathbb{R}^{n}$ that has a homeomorphic copy $A$ of $\mathbb{A}$ as a global attractor. We have shown that to be a global attractor in $\mathbb{R}^{n}$ the set $A$ must be cellular, but cellularity is not a topological property, that is, a priori there is no way to guarantee that $A$ is cellular, even though $\mathbb{A}$ is.

In order to circumvent this problem, we introduce some more refined topological ideas from the theory of shape due to Borsuk [3]. Here we follow Garay [12] and relate trivial shape to contractibility properties (the equivalence of Borsuk's original definition with that given here follows from Borsuk [2] and Hyman [18]).

Definition 3.1. Let $A$ be a subset of $B$. A contraction of $A$ in $B$ is a continuous map

$$
F: A \times[0,1] \rightarrow B
$$

such that $F_{0}=\operatorname{id}_{A}$ and $F_{1} \equiv$ constant map, where $F_{t}$ means the partial map $F_{t}: A \rightarrow B$ given by $F_{t}(p):=F(p, t)$. If such a contraction exists, then we say that $A$ is contractible in $B$. If a set $A$ is contractible in itself, then we simply say that $A$ is contractible.

An easy but important remark is the following.

Remark 3.2. If $A$ is contractible in $B$ and $A_{0} \subseteq A$, then $A_{0}$ is also contractible in $B$. A suitable contraction may be obtained by restricting a contraction of $A$ in $B$. 
As a simple but useful example, we note that any ball $B$ in a normed space is contractible, since there is an obvious contraction onto its centre. Namely, if $B=x_{0}+B_{r}$, then

$$
F(x, t):=x_{0}+(1-t)\left(x-x_{0}\right)
$$

provides a contraction of $B$ in itself.

The following is an extremely trivial proposition, but nevertheless we include it here for comparison purposes with Proposition 3.7.

Proposition 3.3. If $h: A \rightarrow A^{\prime}$ is a homeomorphism and $A$ is contractible, then so is $A^{\prime}$. That is, 'being contractible' is a topological property.

Proof. Let $F: A \times[0,1] \rightarrow A$ be a contraction. Then

$$
h \circ F \circ\left(h^{-1} \times \operatorname{id}_{[0,1]}\right): A^{\prime} \times[0,1] \rightarrow A^{\prime}
$$

is a contraction of $A^{\prime}$.

Now we introduce the property we are interested in, which is weaker than being contractible.

Definition 3.4. Let $X$ be a compact subset of a normed space $E$. We say that $X$ has trivial shape if for every neighbourhood $U$ of $X$ in $E, X$ is contractible in $U$.

Observe that we do not require the existence of a contraction of $X$ in itself, but that there exist contractions of $X$ that take place in arbitrarily small neighbourhoods of $X$ in $E$. When $E$ is a finite-dimensional Euclidean space, a set $X$ having trivial shape is also said to be cell-like.

We now show that strongly cellular sets have trivial shape.

Lemma 3.5. If $E$ is a normed space and $X \subseteq E$ is strongly cellular, then $X$ has trivial shape.

Proof. Since $X$ is strongly cellular, given any neighbourhood $U$ of $X$ we can find a cell $C$ such that $X \subseteq C \subseteq U$. Any ball is contractible, therefore (Proposition 3.3) any cell is contractible. Therefore (Remark 3.2), any subset of a cell is contractible within that cell. Thus $X$ is contractible within $C$, and so within $U$. It follows that $X$ has trivial shape.

The following corollary, an immediate consequence of this result and Lemma 2.2, gives one indication why this definition is potentially interesting.

COROLlary 3.6. If $E$ is a normed space and $\mathbb{A}$ is the global attractor of a homeomorphism $F: E \rightarrow E$, then $\mathbb{A}$ has trivial shape.

However, unlike cellularity, having trivial shape is a topological property.

Proposition 3.7. Let $h: X \rightarrow X^{\prime}$ be a homeomorphism between two compact sets $X$ and $X^{\prime}$ contained in normed spaces $E$ and $E^{\prime}$. Then $X$ has trivial shape if, and only if, $X^{\prime}$ has trivial shape.

Proof. We assume that $X$ has trivial shape. Let $\widehat{h}: E \rightarrow E^{\prime}$ be a continuous extension of $h$; this exists by Dugundji's extension of Tietze's theorem [8].

Let $U^{\prime}$ be a neighbourhood of $X^{\prime}$ in $E^{\prime}$ and consider $U:=\widehat{h}^{-1} U^{\prime}$, which is a neighbourhood of $X$ in $E$. Since $X$ has trivial shape, it is contractible in $U$; let $F: X \times[0,1] \rightarrow U$ be a 
contraction. Then

$$
\widehat{h} \circ F \circ\left(h^{-1} \times \operatorname{id}_{[0,1]}\right): X^{\prime} \times[0,1] \rightarrow U^{\prime}
$$

is a contraction of $X^{\prime}$ in $U^{\prime}$.

One may wonder whether global attractors are actually contractible. The answer is, in general, negative. A quick way to prove this is to observe that a contractible set must be path connected, and then construct examples where global attractors exist that are not path connected. This can be done even in the plane, a good example being the 'topologist's sine curve'

$$
\{(0, y):-1 \leqslant y \leqslant 1\} \cup\{(x, \sin (1 / x)): x \in(0,1]\} \subseteq \mathbb{R}^{2} .
$$

This set is cellular, as can be easily checked from a drawing. Theorem 2.4 guarantees that it is the global attractor of some homeomorphism, but it is not path connected.

\subsection{Global attractors of semiflows}

The following result shows that attractors with trivial shape also arise in more general situations. A semiflow $S(\cdot): E \rightarrow E$ is a family of maps $\{S(t): t \geqslant 0\}$ such that $S(0)$ is the identity map, $S(t+s)=S(t) S(s)$ for all $t, s \geqslant 0$, and $(t, p) \mapsto S(t)(p)$ is continuous.

The definition of a global attractor of a semiflow is an obvious analogue of that of a continuous map.

Proposition 3.8. If $\mathbb{A}$ is the global attractor of a semiflow $S(\cdot)$ on a normed space $E$, then $\mathbb{A}$ has trivial shape.

The result appears in Garay [12], but our proof is much simpler. Note that this result is not a simple consequence of applying Corollary 3.6 to the map $S(T)$ for some fixed $T>0$, since there is no reason why $S(T)$ should be a homeomorphism.

Proof. Let $U$ be a neighbourhood of $\mathbb{A}$. We need to show that $\mathbb{A}$ is contractible in $U$; that is, there exist a continuous map $F: \mathbb{A} \times[0,1] \rightarrow U$ and a point $* \in U$ such that $F(p, 0)=p$ and $F(p, 1)=*$ for every $p \in \mathbb{A}$.

Choose any $q \in \mathbb{A}$ and let $G: \mathbb{A} \times[0,1] \rightarrow E$ be defined as $G(p, t):=q+(1-t)(p-q)$. Clearly, $G$ is a continuous map such that $G(p, 0)=p$ and $G(p, 1)=q$ for every $p \in \mathbb{A}$. Since $\mathbb{A} \times[0,1]$ is compact and $G$ is continuous, its image $C:=G(\mathbb{A} \times[0,1])$ is compact. Thus there exists $T>0$ such that $S(t)(C) \subseteq U$ for every $t \geqslant T$, because $\mathbb{A}$ attracts compact subsets of $E$.

Let $H: \mathbb{A} \times[0,1] \rightarrow U$ be the composition $H:=S(T) \circ G$ (note that the range of $H$ is now $U)$. Denote $*:=S(T)(q)$. Then, since $S(t) \mathbb{A}=\mathbb{A}$ for each $t \geqslant 0$, we have $* \in \mathbb{A} \subseteq U$. Clearly, $H$ is continuous; it also satisfies $H(p, 0)=S(T)(p)$ and $H(p, 1)=*$ for every $p \in \mathbb{A}$. Thus it is almost a contraction of $\mathbb{A}$ in $U$, the only issue being the fact that $H(p, 0)=S(T)(p)$ rather than $H(p, 0)=p$. However, this is easy to fix, as follows. Let $F: \mathbb{A} \times[0,1] \rightarrow U$ be defined as

$$
F(p, t):= \begin{cases}S(2 T t)(p) & \text { if } 0 \leqslant t \leqslant 1 / 2 \\ H(p, 2 t-1) & \text { if } 1 / 2 \leqslant t \leqslant 1\end{cases}
$$

It is straightforward to check that $F$ is continuous and satisfies the required properties $F(p, 0)=p$ and $F(p, 1)=*$ for every $p \in \mathbb{A}$.

We note that in many interesting examples one can show that, at least on the global attractor, the semigroup is injective. In this case, it follows that for any time $t>0$, the time- $t$ map $S(t)$ is a homeomorphism. What follows is therefore applicable to both the global attractors of homeomorphisms, and to the time $t$ map on any global attractor of a semigroup. 


\section{Trivial shape and cellularity in Euclidean spaces}

Now suppose that we begin with a set $\mathbb{A}$ that is the attractor of a homeomorphism (or a semiflow) on a normed space. We therefore know from Corollary 3.6 (or Proposition 3.8) that $\mathbb{A}$ has trivial shape. If $\mathbb{A}$ is finite dimensional, then we can use Theorem 1.1 to find an embedding $e$ : $\mathbb{A} \rightarrow \mathbb{R}^{n}$ for some $n$. Since trivial shape is a topological property (Proposition 3.7), it follows that $e(\mathbb{A}) \subseteq \mathbb{R}^{n}$ has trivial shape.

However, in order to make $e(\mathbb{A})$ the global attractor of a homeomorphism on $\mathbb{R}^{n}$ it must be cellular. We can obtain a cellular set by appealing to the following result due essentially to McMillan [22], but given in precisely the form we require in Daverman [7, III.18, Corollary 5A]. This gives cellularity of $X_{0} \times\{0\}$ in $\mathbb{R}^{n+1}$ whenever $X_{0}$ has trivial shape.

Theorem 4.1 (McMillan-Daverman). If $X_{0}$ is a compact subset of $\mathbb{R}^{n}$ that has trivial shape, then $X_{0} \times\{0\} \subseteq \mathbb{R}^{n+1}$ is cellular.

Daverman includes the hypothesis $n \geqslant 3$ in his statement of the theorem, but the result actually holds for $n \geqslant 1$, since in dimensions 1 and 2 being cellular and having trivial shape are equivalent. For $n=1$, this follows from the fact that a set having trivial shape is connected, so the set $X_{0}$ of Theorem 4.1 must be a compact interval, and then clearly $X_{0} \times\{0\} \subseteq \mathbb{R}^{2}$ is cellular. The case $n=2$ is proved as Corollary $4 \mathrm{C}$ in Section III.15 of Daverman [7].

Corollary 4.2. Let $\mathbb{A}$ be the global attractor of a homeomorphism or a semiflow on a normed space $E$, and assume that $\operatorname{dim}(\mathbb{A}) \leqslant k$. Then there exists a homeomorphism $e: \mathbb{A} \rightarrow$ $X \subseteq \mathbb{R}^{2 k+2}$ such that $X$ is cellular in $\mathbb{R}^{2 k+2}$.

Proof. By Theorem 1.1, there exists a homeomorphism $e: \mathbb{A} \rightarrow X_{0} \subseteq \mathbb{R}^{2 k+1}$. By identifying $\mathbb{R}^{2 k}$ ${ }^{+1}$ with $\mathbb{R}^{2 k+1} \times\{0\} \subseteq \mathbb{R}^{2 k+2}$, we may think of $e$ as a homeomorphism onto the subset $X:=X_{0}$ $\times\{0\} \subseteq \mathbb{R}^{2 k+2}$. Corollary 3.6 or Proposition 3.8 guarantees that the set $\mathbb{A}$ has trivial shape, and the same is true of $X_{0}$ by Proposition 3.7. Then Theorem 4.1 implies that $X$ is cellular in $\mathbb{R}^{2 k+2}$.

\section{Extension of homeomorphisms from compact subsets of $\mathbb{R}^{n}$}

With the above results, we can guarantee that the global attractor $\mathbb{A} \subseteq E$ has trivial shape and find a homeomorphism $e: \mathbb{A} \rightarrow X \subseteq \mathbb{R}^{2 k+2}$ such that $X$ is cellular. Note that the dynamics on $\mathbb{A}$ are reproduced on $X$ by means of the homeomorphism $f=e \circ F \circ e^{-1}$ (the dynamics on $\mathbb{A}$ and $X$ are conjugated by $e$ ).

We now need to extend the homeomorphism $f: X \rightarrow X$ to a homeomorphism on the whole of $\mathbb{R}^{2 k+2}$. Since we have the freedom to increase the dimension of the ambient space (we have done this once already), we can use the elegant trick to due to Klee [19, Statement (3.3)], as outlined in Proposition 5.3.

We make use of the following elementary results on extension of continuous functions.

Lemma 5.1. Let $X \subseteq B_{R} \subseteq \mathbb{R}^{n}$ be a compact set and let $f: X \rightarrow X$ a continuous function. Then there exists a continuous extension $\varphi: \mathbb{R}^{n} \rightarrow \mathbb{R}^{n}$ of $f$ such that $\varphi\left(\bar{B}_{R}\right) \subseteq \bar{B}_{R}$ and $\varphi$ is the identity outside $B_{R}$.

Proof. Let $D$ denote the boundary of $B_{R}$ and extend $f$ to $X \cup D$ by letting it be the identity on $D$ (this is still a continuous map). Considering this as a map from $X \cup D$ into $\bar{B}_{R}$, the Tietze extension theorem can be used to obtain a continuous $\varphi: \bar{B}_{R} \rightarrow \bar{B}_{R}$ such that $\left.\varphi\right|_{X}=f$ and $\left.\varphi\right|_{D}=$ id. It remains only to set $\varphi(x):=x$ for $x \notin B_{R}$. 
The second simple lemma will be crucial in obtaining a controlled extension in the subsequent theorem.

Lemma 5.2. Let $X \subseteq B_{R} \subseteq \mathbb{R}^{n}$ be a compact set. Then there exists a homeomorphism $c: \mathbb{R}^{n} \rightarrow \mathbb{R}^{n}$ such that $\left.c\right|_{X \cup\{0\}}=\operatorname{id}_{X \cup\{0\}}$ and $c\left(B_{r}\right) \subseteq B_{r / 2}$ for every $r \geqslant 2 R$.

Proof. Choose $R^{*}<R$ be so close to $R$ that $X \subseteq B_{R^{*}}$. Let $\theta:[0, \infty) \rightarrow[0, \infty)$ be the (unique) continuous map that is affine on each of the intervals $\left[0, R^{*}\right],\left[R^{*}, 2 R\right]$, and $[2 R, \infty)$ and such that (i) $\theta(r)=r$ for $0 \leqslant r \leqslant R^{*}$, (ii) $\theta(r)=r / 2$ for $r \geqslant 2 R$. Note that $\theta$ is strictly increasing and surjective, hence a homeomorphism.

Define $c: \mathbb{R}^{n} \rightarrow \mathbb{R}^{n}$ by

$$
c(p):= \begin{cases}0 & \text { for } p=0 \\ \theta(|p|) p /|p| & \text { for } p \neq 0 .\end{cases}
$$

Clearly, $c$ is continuous except possibly at $p=0$. However, for $p \in B_{R^{*}}$ we have $c(p)=p$, s o $\quad c$ is continuous at $p=0$ too. This also shows that $\left.c\right|_{X}=\mathrm{i} \mathrm{d}{ }_{X}$. Replacing $\theta$ in (5.1) b y $\theta^{-1}$ yields an expression for $c^{-1}$, which is thus also continuous, and hence $c$ is a homeomorphism.

It remains only to show that $c\left(B_{r}\right) \subseteq B_{r / 2}$ for every $r \geqslant 2 R$. Thus, let $r \geqslant 2 R$ and pick $p \in B_{r}$. If $|p| \leqslant 2 R$, then $\theta(|p|) \leqslant R$ and so $c(p) \in B_{R} \subseteq B_{r / 2}$. If $|p|>2 R$, then $\theta(|p|)=|p| / 2<r / 2$, so $c(p) \in B_{r / 2}$.

We now prove our controlled extension theorem.

Proposition 5.3. Let $X \subseteq B_{R} \subseteq \mathbb{R}^{n}$ be a compact set and let $f: X \rightarrow X$ a homeomorphism. Then there exists a homeomorphism $\hat{f}: \mathbb{R}^{2 n} \rightarrow \mathbb{R}^{2 n}$ such that

$$
\hat{f}\left(x, 0_{n}\right)=\left(f(x), 0_{n}\right) \quad \text { for all } x \in X,
$$

where $0_{n}$ is the origin in $\mathbb{R}^{n}$, and such that

$$
(x, y) \in B_{r} \times B_{r} \Rightarrow \hat{f}(x, y) \in B_{r} \times B_{r}
$$

for any $r \geqslant R$.

Proof. We use Lemma 5.1 to extend the continuous map $f: X \rightarrow \mathbb{R}^{n}$ to a continuous map $\varphi$ : $\mathbb{R}^{n} \rightarrow \mathbb{R}^{n}$ that is the identity outside $B_{R}$ and for which $\varphi\left(B_{R}\right) \subseteq B_{R}$, and again to extend the continuous map $f^{-1}: X \rightarrow \mathbb{R}^{n}$ to another continuous map $\psi: \mathbb{R}^{n} \rightarrow \mathbb{R}^{n}$ that is the identity outside $B_{R}$ and for which $\psi\left(\bar{B}_{R}\right) \subseteq \bar{B}_{R}$.

Let $f_{1}, f_{2}: \mathbb{R}^{2 n} \rightarrow \mathbb{R}^{2 n}$ be the homeomorphisms defined by

$$
f_{1}(x, y)=(x, y+\varphi(x)) \quad \text { and } \quad f_{2}(x, y)=(2 y+\psi(x), x)
$$

for $x, y \in \mathbb{R}^{n}$. One can check that these are homeomorphisms since their inverses are given explicitly by

$$
f_{1}^{-1}(x, y)=(x, y-\varphi(x)) \quad \text { and } \quad f_{2}^{-1}(x, y)=(y,(x-\psi(y)) / 2) .
$$


Define $g=f_{2}^{-1} \circ f_{1}$; this is a homeomorphism of $\mathbb{R}^{2 n}$ since it is the composition of homeomorphisms. Note that for every $x \in X$

$$
f_{1}(x, 0)=(x, \varphi(x))=(x, f(x))
$$

and

$$
f_{2}(f(x), 0)=(\psi(f(x)), f(x))=(x, f(x)),
$$

so

$$
g(x, 0)=\left(f_{2}^{-1} \circ f_{1}\right)(x, 0)=f_{2}^{-1}(x, f(x))=(f(x), 0) .
$$

The argument to this point provides a homeomorphism that extends $f$. We now combine this with the homeomorphism of Lemma 5.2 to obtain the controlled extension we require.

Let $c: \mathbb{R}^{n} \mathbb{R}^{n}$ be the homeomorphism constructed in Lemma 5.2 , which gives rise to another

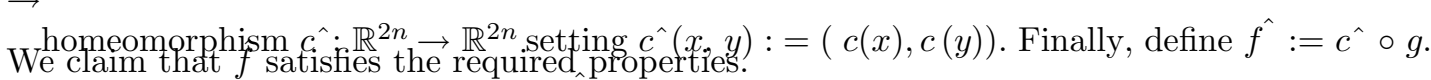

Since $\left.\hat{c}\right|_{X \times\{0\}}=\mathrm{id}_{X \times\{0\}}$, clearly $\hat{f}(x, 0)=(f(x), 0)$ for $x \in X$. Now let $r \geqslant R$ and pick $(x, y) \in B_{r} \times B_{r}$. Then $|\varphi(x)| \leqslant r$, so

$$
\left(x^{\prime}, y^{\prime}\right)=f_{1}(x, y)=(x, y+\varphi(x)) \in B_{r} \times B_{2 r},
$$

and similarly $\left|\psi\left(y^{\prime}\right)\right| \leqslant 2 r$, so

$$
g(x, y)=f_{2}^{-1}\left(x^{\prime}, y^{\prime}\right)=\left(y^{\prime},\left(x^{\prime}-\psi\left(y^{\prime}\right)\right) / 2\right) \in B_{2 r} \times B_{2 r} .
$$

Now $2 r \geqslant 2 R$ so $c\left(B_{2 r}\right) \subseteq B_{r}$, and hence $\hat{f}(x, y)=\hat{c}(g(x, y)) \in B_{r} \times B_{r}$.

REMARK 5.4. In the applications of Proposition 5.3, it would suffice for the condition $\hat{f}\left(B_{r} \times\right.$ $\left.B_{r}\right) \subseteq B_{r} \times B_{r}$ of (5.2) to hold only for a restricted set of radii, for example, those of the form $r$ $=2{ }^{j} R$ with $j \geqslant 0$.

It is natural to ask whether the doubling of the dimension used in Proposition 5.3 is really required. Certainly one cannot, in general, extend a homeomorphism $f: X \rightarrow X$, where $X$ is a compact subset of $\mathbb{R}^{n}$, to a homeomorphism on the whole of $\mathbb{R}^{n}$. For instance, let $X \subseteq \mathbb{R}^{2}$ be the union of two concentric circumferences and let $f: X \rightarrow X$ be a homeomorphism that interchanges the two connected components of $X$. Then $f$ cannot be extended to a homeomorphism of all of $\mathbb{R}^{2}$ because such an extension would have to send one of the bounded components of $\mathbb{R}^{2} \backslash X$ onto the unbounded one, which is impossible. This same example can be turned into a connected set by joining the two circumferences by a radial segment lying between them.

In the application of Proposition 5.3 to our dynamical problem in the next section, $X$ will be cellular. Moreover, if the original $F$ that generates the dynamics on $E$ is the time-one map of a flow, then $\left.F\right|_{\mathbb{A}}: \mathbb{A} \rightarrow \mathbb{A}$ is isotopic to the identity and the same is true of $f: X \rightarrow X$. I n this case, a (hard) theorem of Oversteegen and Tymchatyn [26] shows that $f$ can indeed be extended to a homeomorphism of all of $\mathbb{R}^{2}$.

However, in dimensions 3 and higher, even in the favourable case just mentioned that $X$ is cellular and $f$ is isotopic to the identity, extending $f$ to a homeomorphism of all $\mathbb{R}^{n}$ may not be possible. To illustrate this point, we shall consider the $\operatorname{arc} A \mathrm{~s} \mathrm{~h}$ o w n i n F i g u r e 1 (a detailed description can be found in [24]). It is the union of two subarcs $A_{1}$ and $A_{2}$, the first consisting of infinitely many trefoil knots that accumulate at one of its endpoints $p$ and the second being just a straight line segment $p q$.

It is easy to see from the drawing that: (i) $A$ is cellular and (ii) each point of the arc other than $p$ has a neighbourhood $U$ in $\mathbb{R}^{3}$ such that there exists a homeomorphism of $\mathbb{R}^{3}$ that sends $U \cap A$ into a straight line segment ( $A$ is said to be 'locally polyhedral' at these 


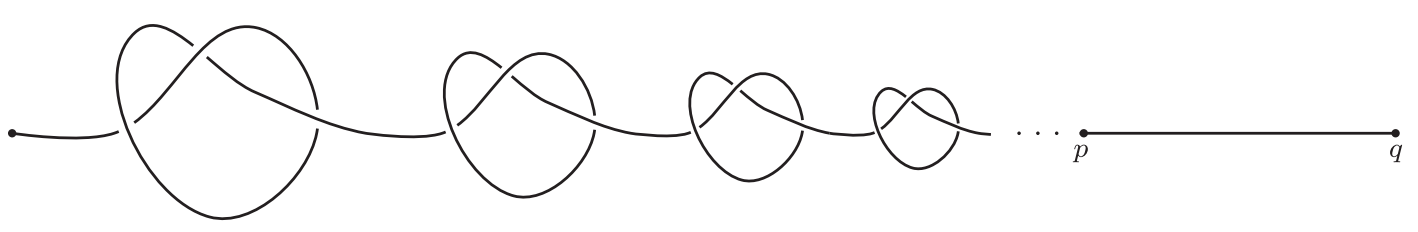

Figure 1. The arc $A$.

points). Although more difficult to prove (we refer the reader again to [24]), it can be shown that (iii) $p$ does not have this property. Also note that, by definition, (iv) if $h: \mathbb{R}^{3} \rightarrow \mathbb{R}^{3}$ is a homeomorphism such that $h(A)=A$ and the arc is locally polyhedral at a point $p^{\prime}$, then it is also locally polyhedral at $h\left(p^{\prime}\right)$ (that is, being locally polyhedral at a point is preserved under ambient homeomorphisms leaving $A$ invariant).

Now let $X$ be the arc $A$ and let $f: X \rightarrow X$ a homeomorphism such that $f(p) \neq p$. For instance, if we think of the arc as being parametrised by some homeomorphism $\alpha:[0,1] \longrightarrow A$, we could choose $f$ to send $\alpha(t)$ onto $\alpha\left(t^{2}\right)$. This $f$ is isotopic to the identity and its only fixed points are the endpoints of the arc. If $f$ could be extended to a homeomorphism $\hat{f}: \mathbb{R}^{3} \rightarrow \mathbb{R}^{3}$, by (ii)-(iv) above necessarily $\hat{f}(p)=p$, contradicting the condition that $f(p) \neq p$. Thus we have exhibited a cellular compactum $X \subseteq \mathbb{R}^{3}$ and a homeomorphism $f$ of $X$ (isotopic to the identity) that has no extension to a homeomorphism of $\mathbb{R}^{3}$.

\section{Finite-dimensional dynamics}

Finally, we can combine these ingredients to show that the dynamics on $\mathbb{A}$ occur within the global attractor of a finite-dimensional system.

TheOREM 6.1. Let $\mathbb{A}$ be the global attractor of a homeomorphism $F: E \rightarrow E$ with $\operatorname{dim}(\mathbb{A}) \leqslant k$. For any $\epsilon>0$, there exist homeomorphisms

$$
e: \mathbb{A} \rightarrow A \subseteq \mathbb{R}^{4 k+4} \quad \text { and } \quad f: \mathbb{R}^{4 k+4} \rightarrow \mathbb{R}^{4 k+4}
$$

such that $A$ is invariant under $f$ and the dynamics on $\mathbb{A}$ induced by $F$ and the dynamics on $A$ induced by $f$ are conjugate under $e$, that is,

$$
\left.F\right|_{\mathbb{A}}=e^{-1} \circ f \circ e,
$$

and such that $f$ has a global attractor $A_{f}$ with

$$
A \subseteq A_{f} \subseteq N(A, \epsilon)
$$

where

$$
N(A, \epsilon)=\left\{y \in \mathbb{R}^{4 k+4}: \operatorname{dist}(y, A)<\epsilon\right\} .
$$

In other words, $A \simeq \mathbb{A}$ is 'almost' the global attractor of $f$; and the dynamics of $\left.F\right|_{\mathbb{A}}$ are no more complicated than those of $f$.

Proof. Corollary 4.2 provides a homeomorphism $e: \mathbb{A} \rightarrow X_{1} \subseteq \mathbb{R}^{2 k+2}$ where $X_{1}$ is cellular in $\mathbb{R}^{2 k}$ +2 . Using Theorem 2.4, we may find a homeomorphism $h: \mathbb{R}^{2 k+2} \rightarrow \mathbb{R}^{2 k+2}$ such that $X_{1}$ is the global attractor of $h$ and $\left.h\right|_{X_{1}}=\mathrm{id}$. C h o o s e $R$ such that $X_{1} \subseteq B_{R}$. Then we can assume that there is a constant $\rho>0$ such that $h\left(B_{r}\right) \subseteq B_{r-\rho}$ for $r \geqslant R$ and, in particular, $h^{m}\left(B_{r}\right) \subseteq$ $B_{\max (r-m \rho, R)}$ for all $m \geqslant 1$ a n d $r>0$. 
The map $f_{1}: X_{1} \rightarrow X_{1}$ defined by $f_{1}(x)=e \circ F \circ e^{-1}(x)$ is a homeomorphism. We use Proposition 5.3 to produce a homeomorphism $\hat{\hat{f}_{1}}: \mathbb{R}^{4 k+4} \rightarrow \mathbb{R}^{4 k+4}$ such that

$$
\hat{f}_{1}(x, 0)=\left(f_{1}(x), 0\right) \text { for all } x \in X_{1},
$$

that is, $\hat{f}_{1}$ extends $f_{1}$ from the set $A:=X_{1} \times\left\{0_{2 k+2}\right\}$ to all of $\mathbb{R}^{4 k+4}$. We can choose $\hat{f}_{1}$ so that

$$
(x, y) \in B_{r} \times B_{r} \Rightarrow \hat{f}_{1}(x, y) \in B_{r} \times B_{r}
$$

for any $r \geqslant R$.

Consider the homeomorphism $\hat{h}: \mathbb{R}^{4 k+4} \rightarrow \mathbb{R}^{4 k+4}$ given by $\hat{h}(x, y):=(h(x), y / 2)$ for $x, y \in \mathbb{R}^{2 k+2}$. Clearly, $A$ is a global attractor for $\hat{h}$ and $\left.\hat{h}\right|_{A}=$ id. Let $K=\bar{B}_{R} \times \bar{B}_{R}$, and choose so large an $m \geqslant 1$ that $\hat{h}^{m}(K) \subseteq N(A, \epsilon)$. Set $f:=\hat{h}^{m} \circ \hat{f}_{1}$. We claim that $f$ satisfies the required properties.

It is clear that $\left.f\right|_{A}=\left.f_{1}\right|_{A}$, since $\hat{h}$ is the identity on $A$. Thus $A$ is a compact invariant set under $f$, and $e^{-1} \circ f \circ e=\left.F\right|_{\mathbb{A}}$. We show that $K$ is an attracting set for $f$. Then $f$ has a global attractor $A_{f}$ by Theorem 2.1, a n d $A \subseteq A_{f} \subseteq K$. M o r e o v e r ,

$$
A_{f}=f\left(A_{f}\right) \subseteq f(K)=\hat{h}^{m} \hat{f}_{1}(K) \subseteq \hat{h}^{m}(K) \subseteq N(A, \epsilon) .
$$

To show that $K$ is an attracting set, consider the mapping $\phi(r):=\max \left(r-m \rho, r / 2^{m}, R\right)$. We claim that

$$
f\left(B_{r} \times B_{r}\right) \subseteq B_{\phi(r)} \times B_{\phi(r)} \quad \text { for } r>0 .
$$

This is easily seen to be true for $r \leqslant R$ because

$$
f\left(B_{R} \times B_{R}\right) \subseteq B_{R} \times B_{R},
$$

while for $r>R$ we have

$$
\hat{f}_{1}\left(B_{r} \times B_{r}\right) \subseteq B_{r} \times B_{r}
$$

and then

$$
\hat{h}^{m} \hat{f}_{1}\left(B_{r} \times B_{r}\right) \subseteq B_{\max (r-m \rho, R)} \times B_{r / 2^{m}} \subseteq B_{\phi(r)} \times B_{\phi(r)} .
$$

Now let $C$ be a bounded subset of $\mathbb{R}^{4 k+4}$ and choose $r$ such that $C \subseteq B_{r} \times B_{r}$. For $n$ sufficiently large $\phi^{n}(r)=R$, in which case

$$
f^{n}(C) \subseteq f^{n}\left(B_{r} \times B_{r}\right) \subseteq B_{\phi^{n}(r)} \times B_{\phi^{n}(r)}=B_{R} \times B_{R},
$$

more than is required.

\section{Conclusion and open problems}

Theorem 6.1 shows that the dynamics on a finite-dimensional global attractor of a homeomorphism are no more complicated than the dynamics that can arise in a finite-dimensional system.

However, it is natural to wonder whether it would in fact be possible to construct a homeomorphism $f: \mathbb{R}^{4 k+4} \rightarrow \mathbb{R}^{4 k+4}$ such that $A_{f}=A$ in Theorem 6.1 , that is, finite-dimensional global attractors of homeomorphisms can always be realised, along with their dynamics, as global attractors in finite-dimensional spaces. Even if one allows the finite-dimensional map to be continuous rather than a homeomorphism, to our knowledge this problem is still open.

We can reformulate this problem in a more topological way. Suppose that $X \subseteq B_{R}$ is a cellular subset of $\mathbb{R}^{n}$ written as the intersection of the cellular sequence $\left(C_{j}\right)$,

$$
X=\bigcap_{j=1}^{\infty} C_{j} .
$$


Also, let a homeomorphism $f: X \rightarrow X$ be given. Proposition 5.3 is a controlled extension result: it provides a homeomorphism $\hat{f}: \mathbb{R}^{2 n} \rightarrow \mathbb{R}^{2 n}$ that extends $f$ (in the sense that $\hat{f}\left(x, 0_{n}\right)=$ $\left(f(x), 0_{n}\right)$ for every $\left.x \in X\right)$ and such that

$$
\hat{f}\left(B_{r} \times B_{r}\right) \subseteq B_{r} \times B_{r}
$$

for every $r \geqslant R$. We are now going to show that if the extension $\hat{f}$ can also be controlled near $X$ (and not only for $r \geqslant R$ ), then it is possible to achieve $A_{f}=A$ in Theorem 6.1.

Proposition 7.1. Suppose that Proposition 5.3 could be strengthened so that, in addition to (7.1) ( possibly weakened as in Remark 5.4), the relation

$$
\hat{f}\left(C_{j} \times \bar{B}_{R / 2^{j-1}}\right) \subseteq C_{j} \times \bar{B}_{R / 2^{j-1}}
$$

held for every $j \geqslant 1$. Then in Theorem 6.1 we could achieve $A_{f}=A$. That is, if $\mathbb{A}$ is the global attractor of a homeomorphism $F: E \rightarrow E$ with $\operatorname{dim}(\mathbb{A}) \leqslant k$, then there exist homeomorphisms

$$
e: \mathbb{A} \rightarrow A \subseteq \mathbb{R}^{4 k+4} \text { and } f: \mathbb{R}^{4 k+4} \rightarrow \mathbb{R}^{4 k+4}
$$

such that the dynamics on $\mathbb{A}$ and $A$ are conjugate under $e$, and $A$ is the global attractor of $f$.

Proof. Let $e, X_{1}, B_{R}, h, f_{1}, \hat{h}$, and $K$ be as in the proof of Theorem 6.1. We have $h\left(\bar{B}_{R}\right) \subseteq$ $\bar{B}_{R-\rho}$ for some $\rho>0$ so $h\left(\bar{B}_{R}\right) \subseteq B_{R}$. For each $j=1,2, \ldots$ let

$$
C_{j}=h^{j-1}\left(\bar{B}_{R}\right)
$$

clearly $\left(C_{j}\right)$ is a cellular sequence for $X_{1}$. Now pick an extension $\hat{f_{1}}: \mathbb{R}^{4 k+4} \rightarrow \mathbb{R}^{4 k+4}$ that satisfies both (7.1) and (7.2) i n p l a c e of $f^{\wedge}$ (and with $\left.n=2 k+2\right)$. Define $f:=h \circ \hat{f^{\prime}}{ }_{1}$. The same argument as that given in Theorem 6.1 proves that for any bounded set $C$ we have $f^{n}(C) \subseteq K$ for all sufficiently large $n$, s o $f$ has a global attractor $A_{f} \subseteq K$. Clearly, $A \subseteq A_{f}$. M o r e o v e r, observe that

$$
f\left(C_{j} \times \bar{B}_{R / 2^{j-1}}\right)=\hat{h}\left(\hat{f}_{1}\left(C_{j} \times \bar{B}_{R / 2^{j-1}}\right)\right) \subseteq \hat{h}\left(C_{j} \times \bar{B}_{R / 2^{j-1}}\right)=C_{j+1} \times \bar{B}_{R / 2^{j}}
$$

for every $j \geqslant 1$, so

$$
f^{n}\left(C_{j} \times \bar{B}_{R / 2^{j-1}}\right) \subseteq C_{j+n} \times \bar{B}_{R / 2^{j+n-1}}
$$

for all $j \geqslant 1$ and $n \geqslant 1$. Now, for every $n \geqslant 1$ the case $j=1$ yields

$$
f^{n}(K)=f^{n}\left(C_{1} \times \bar{B}_{R}\right) \subseteq C_{n+1} \times \bar{B}_{R / 2^{n}} .
$$

By Theorem 2.1, we get

$$
A_{f}=\bigcap_{n=1}^{\infty} f^{n}(K) \subseteq \bigcap_{n=1}^{\infty}\left(C_{n+1} \times \bar{B}_{R / 2^{n}}\right)=X_{1} \times\{0\}=A,
$$

as required.

In view of the usefulness of having a controlled extension result that satisfies both (7.1) a n d (7.2), we restate it more formally as an open question.

QUeSTION 7.2. Let $X \subseteq B_{R} \subseteq \mathbb{R}^{n}$ be a compact set, let $f: X \rightarrow X$ a homeomorphism, and let $\left(C_{j}\right)$ a cellular sequence for $X$. Does there exist a homeomorphism $\hat{f}: \mathbb{R}^{2 n} \rightarrow \mathbb{R}^{2 n}$ such that

$$
\hat{f}\left(x, 0_{n}\right)=\left(f(x), 0_{n}\right) \quad \text { for all } x \in X,
$$

where $0_{n}$ is the origin in $\mathbb{R}^{n}$;

$$
\hat{f}\left(B_{2^{j} R} \times B_{2^{j} R}\right) \subseteq B_{2^{j} R} \times B_{2^{j} R}
$$


for every $j \geqslant 0$; and

$$
\hat{f}\left(C_{j} \times \bar{B}_{R / 2^{j-1}}\right) \subseteq C_{j} \times \bar{B}_{R / 2^{j-1}}
$$

for every $j \geqslant 1$ ?

As remarked in Section 1, in the case of semiflows the problem of embedding the attractor along with its dynamics seems much more difficult and is still entirely open.

Acknowledgements. We would like to thank the referee for two extremely close readings of the paper, which eliminated many small errors and inconsistencies, and provided many suggestions which have improved the clarity of the presentation, in particular simplification of the proof of Theorem 6.1 and a sharpening of Question 7.2.

\section{References}

1. A. V. BAbin and M. I. Vishik, Attractors of evolution equations (North-Holland Publishing, Amsterdam, 1992).

2. K. Borsuk, 'A note on the theory of shape of compacta', Fund. Math. 67 (1970) 265-278.

3. K. Borsuk, Theory of shape (PWN, Warsaw, 1975).

4. M. Brown, 'A proof of the generalized Schoenflies theorem', Bull. Amer. Math. Soc. 66 (1960) 74-76.

5. V. V. Chepyzhov and M. I. Vishik, Attractors for equations of mathematical physics (American Mathematical Society, Providence, RI, 2002).

6. I. D. Chueshov, Introduction to the theory of infinite-dimensional dissipative systems (University Lectures in Contemporary Mathematics, ACTA, Kharkiv, 2002).

7. R. J. Daverman, Decompositions of manifolds (Academic Press, Orlando, FL, 1986).

8. J. Dugundu, 'An extension of Tietze's theorem', Pacific J. Math. 1 (1951) 353-367.

9. A. Eden, C. Foias, B. Nicolaenko and R. Temam, Exponential attractors for dissipative evolution equations (Wiley, Chichester, 1994).

10. A. Eden, V. K. Kalantarov and S. V. Zelik, 'Counterexamples to regularity of Ma né projections in the theory of attractors', Russian Math. Surveys 68 (2013) 199-226.

11. C. Foias, G. R. Sell and R. Temam, 'Inertial manifolds for nonlinear evolutionary equations', J. Differential Equations 73 (1988) 309-353.

12. B. M. Garay, 'Strong cellularity and global asymptotic stability', Fund. Math. 138 (1991) 147-154.

13. B. Günther, 'Construction of differentiable flows with prescribed attractor', Topology Appl. 62 (1995) 87-91.

14. B. Günther and J. SEgal, 'Every attractor of a flow on a manifold has the shape of a finite polyhedron', Proc. Amer. Math. Soc. 119 (1993) 321-329.

15. J. K. Hale, Asymptotic behavior of dissipative systems (American Mathematical Society, Providence, RI, 1988).

16. J. Heinonen, Geometric embeddings of metric spaces, vol. 90 (Report University of Jyväskylä Department of Mathematics and Statistics, Jyväskylä, 2003).

17. W. Hurewicz and H. Wallman, Dimension theory (Princeton University Press, Princeton, NJ, 1941).

18. D. M. Hyman, 'On decreasing sequences of compact absolute retracts', Fund. Math. 64 (1969) 91-97.

19. V. L. KleE JR., 'Some topological properties of convex sets', Trans. Amer. Math. Soc. 78 (1955) 30-45.

20. O. A. Ladyzhenskaya, Attractors for semigroups and evolution equations (Cambridge University Press, Cambridge, UK, 1991).

21. R. A. MCCoy, 'Cells and cellularity in infinite-dimensional normed linear spaces', Trans. Amer. Math. Soc. 176 (1973) 401-410.

22. D. R. McMillan JR., 'A criterion for cellularity in a manifold', Ann. of Math. (2) 79 (1964) 327-337.

23. K. Menger, 'Über umfassendste n-dimensionale Mengen', Proc. Akad. Wetensch. Amst. 29 (1926) 1125-1128.

24. E. E. Moise, Geometric topology in dimensions 2 and 3 (Springer, New York, 1977).

25. G. NöBELING, 'Über eine $n$-dimensionale Universalmenge im $R^{2 n+1}$ ', Math. Ann. 104 (1931) 71-80.

26. L. G. Oversteegen and E. D. Tymchatyn, 'Extending isotopies of planar continua', Ann. of Math. (2) 172 (2010) 2105-2133.

27. E. Pinto de Moura, J. C. Robinson and J. J. SÁnchez-Gabites, 'Embedding of global attractors and their dynamics', Proc. Amer. Math. Soc. 139 (2011) 3497-3512.

28. J. C. Robinson, 'Global attractors: topology and finite-dimensional dynamics', J. Dynam. Differential Equations 11 (1999) 557-581.

29. J. C. Robinson, Infinite-dimensional dynamical systems (Cambridge University Press, Cambridge, UK, 2001). 
30. J. C. Robinson, Dimensions, embeddings, and attractors (Cambridge University Press, Cambridge, UK, 2011).

31. A. V. Romanov, 'Finite-dimensional limiting dynamics of dissipative parabolic equations', Sb. Math. 191 (2000) 415-429.

32. R. Temam, Infinite-dimensional dynamical systems in mechanics and physics (Springer, New York, 1988).

James C. Robinson

Mathematics Institute

University of Warwick

Coventry

CV4 7AL

United Kingdom

j.c.robinson@warwick.ac.uk
Jaime J. Sánchez-Gabites

Departamento de Análisis Económico: Métodos Cuantitativos

Facultad de Ciencias Económicas y

Empresariales

Universidad Autónoma de Madrid

28049 Cantoblanco (Madrid)

Spain

jaimej.sanchez@uam.es 